\title{
What Health Care Providers Know and How They Communicate Breast Cancer Risk to Patients
}

\section{Authors:}

Marlyn Allicock ${ }^{\mathbf{1}}, \mathrm{PhD}, \mathrm{MPH}$

Kathleen Gray ${ }^{2}$, MSPH

Neasha Graves ${ }^{2}$, MPA

Liza Makowski ${ }^{2}, \mathrm{PhD}$

Shannon A. Allport ${ }^{1}$, BA

Kenda Freeman ${ }^{2}$, MPH

Melissa A. Troester ${ }^{2}, \mathrm{PhD}$

Authors' affiliations:

1 University of Texas, School of

Public Health

2 University of North Carolina at Chapel Hill

\section{Corresponding Author:}

Marlyn Allicock, PhD, MPH

E-mail:

Marlyn.A.Allicock@uth.tmc.edu

The University of Texas School of

Public Health

\section{Authors' emails:}

Marlyn Allicock

Marlyn.A.Allicock@uth.tmc.edu

Kathleen Gray

kmgray@email.unc.edu

Neasha Graves

neasha_graves@unc.edu

Liza Makowski

liza.makowski@unc.edu

Shannon A. Allport

Shannon.A.Allport@uth.tmc.edu

Kenda Freeman

ktfreeman@live.unc.edu

Melissa A. Troester

troester@unc.edu

\begin{abstract}
Racial disparities in breast cancer mortality continue, partly due to higher prevalence of an aggressive breast cancer subtype called basallike breast cancer (BBC) in African-Americans. Health care providers (HCPs) are uniquely positioned to discuss cancer risk and prevention with patients. We investigated breast cancer knowledge and risk communication among HCPs to identify factors that influenced communication with patients. Interviews were conducted with 34 HCPs in North Carolina. We found limited evidence of breast cancer risk education, and specific subtypes of breast cancer were not discussed. Barriers to communication about prevention include limited time, perceived patient receptivity and education level, and scientific misinformation. Factors that prompted discussions included patient characteristics (age, race, and socioeconomic status). To broaden the conversation, HCPs must receive and communicate more accurate information on breast cancer risk. Given these barriers to breast cancer education, additional opportunities to intervene with high-risk populations must be identified.
\end{abstract}

\section{Keywords:}

breast cancer, basal-like breast cancer, health communication, risk factors, health disparity

Word count: 3917 


\section{INTRODUCTION}

Breast cancer is the most common type of cancer among women. ${ }^{1}$ Risk reduction strategies include lifestyle modification, genetic counseling, and screening for women at risk for hereditary breast cancer, as well as chemoprevention. ${ }^{2-5}$ Although information about breast cancer risks is commonly available, many women still misperceive their own risk and cannot pinpoint major risk factors. ${ }^{6}$ Health care providers (HCPs) in primary care settings are uniquely positioned to discuss breast cancer risk and risk reduction with their patients to reduce disease burden. Effective communication between HCPs and patients is a key factor influencing patient behavior and well-being. ${ }^{7-11}$ Quality communication at the phases of disease prevention, diagnosis, treatment, and end-of-life require mutual understanding and informed decision-making, and lead to more effective health care delivery and improved health outcomes. $^{12,13}$

Poor patient-provider communication has been suggested as one mechanism underlying racial and ethnic health disparities. $^{14,15}$ Both patient and HCP behaviors have been studied to assess how communication influences patient outcomes. ${ }^{16,17}$ However, because morbidity and mortality disparities between minorities and Whites continue to exist in the case of breast cancer, it is critical to have a greater understanding of unique considerations for these minority groups and how both barriers and facilitators to HCP communication with minorities affect health outcomes.

African-Americans have the highest mortality and lowest survival rate of any racial and ethnic group with regards to alltypes of cancer, and also for breast cancer in particular. ${ }^{1}$ Furthermore, the highly aggressive basal-like breast cancer (BBC) subtype affects African-Americans disproportio- nately. ${ }^{18}$ BBC has a unique risk factor profile, in striking contrast to other forms of breast cancer. These complexities in risk factor patterns for basal-like versus other breast cancer subtypes have created important risk communication challenges for HCPs in primary care settings.

Despite these challenges, viable BBC prevention strategies have been proposed. It has been estimated that $68 \%$ of BBC could be prevented by reducing obesity and increasing breastfeeding. ${ }^{19}$ Because AfricanAmericans have higher rates of obesity, ${ }^{20}$ gain and retain more weight with pregnancy, $^{21}$ and have lower rates of breastfeeding, ${ }^{22}$ HCPs are in a unique position to disseminate the importance of these health behaviors in reducing risk of not only $\mathrm{BBC}$, but other types breast cancer as well.

This study used in-depth interviews with 34 health professionals to characterize how knowledgeable providers were about (1) breast cancer risk overall, and (2) BBC subtype-specific risk factors. We also sought to identify factors that influenced HCPs discussions of breast cancer risk, prevention, and screening information with patients.

\section{METHODS}

2.1 Study design and sampling methodology. A qualitative study was conducted in 2011 using semi-structured interviews with 34 HCPs - family physicians, physician assistants (PAs), nurse practitioners (NPs), registered nurses (RNs), health educators, and registered dieticians in primary care services. We recruited HCPs through letters, emails, and phone calls to outpatient clinics, community health centers, health departments, area hospitals, and professional organizations in central North Carolina. Eligibility for participation included current HCPs employed in central North Carolina and serving female patients. 
To avoid selection bias due to small samples in qualitative studies, a diversity of profiles was guaranteed by recruiting HCPs in both urban and rural communities, in a variety of different clinical settings, and by selecting multiple participants to represent each HCP profession listed above. Interested individuals contacted the study coordinator and completed screening questions; if they were determined to be eligible, they were invited to participate. Thirty-four HCPs consented to be interviewed. Interviews were conducted in-person, unless requested by telephone. All study procedures were approved by the institutional review board at the University of North Carolina at Chapel Hill (IRB\# 10-2157).

2.2 Data collection. Interviews with the 34 participants were conducted in 2011. The research team developed an interview guide to provide consistency in questioning, but with latitude to probe individual responses for more detail if necessary. The interviews covered several topics: 1) concerns about general patient health issues; 2) breast cancer risk (perceptions of patient knowledge regarding risk, frequency discussing risk, tools used to educate about risk, barriers to conveying information); 3 ) communication with patients about breast cancer; and 4) use of technical terminology with patients. HCPs were also administered a questionnaire that included a series of questions to assess more specific knowledge about breast cancer terms and subtypes (e.g. $\mathrm{BBC}$, breast cancer subtypes, clinical trials for cancer, triple negative breast cancer, and targeted therapy). Additionally, if HCPs responded "yes" to the terms, they were further queried on their use of the terms with patients, including frequency and context within which they used the terms. Given that physician knowledge about breast cancer terms has been well characterized previously ${ }^{23}$, we posed specific breast cancer terminology questions to dietitians, nurses, nurse practitioners, and health educators only. All interviews were conducted by the same research team member, audio-taped with the HCPs' consent, and lasted approximately 40-60 minutes. Written consent was obtained from all study participants prior to the interviews. Physicians, PAs, and NPs were compensated $\$ 100$, and all others received $\$ 40$ for participating.

2.3 Analysis. Data were transcribed verbatim, coded, and analyzed for frequencies of relevant content themes. Thematic content analysis was used, which uses an inductive approach to identify common provider perceptions of their patients' needs for information about breast health, to discover how HCPs communicate to patients about breast cancer risk, and to identify barriers to communication as they emerged from the data. ${ }^{24}$ Three investigators independently reviewed transcripts and met with all authors to discuss emerging themes and coding strategies to guide text abstraction. Broad themes were created after each team member read the transcripts and were refined during the initial coding process. Results were organized by theme and subcategory and quotes describing the themes were extracted. 
Medical Research Archives, Vol. 5, Issue 7, July 2017

PROVIDER COMMUNCATION AND BREAST CANCER RISK

Table 1

Healthcare Provider Demographics $(\mathrm{n}=34)$

\begin{tabular}{|c|c|}
\hline Characteristic & $\%(\mathrm{n})$ \\
\hline \multicolumn{2}{|l|}{ Occupation } \\
\hline Dietitian & $6 \%(2)$ \\
\hline Health Educator & $26 \%(9)$ \\
\hline Nurse & $32 \%(11)$ \\
\hline Nurse Practitioner & $6 \%(2)$ \\
\hline Physician & $18 \%(6)$ \\
\hline Physician Assistant & $12 \%(4)$ \\
\hline \multicolumn{2}{|l|}{ Age, years } \\
\hline $18-29$ & $15 \%(5)$ \\
\hline $30-39$ & $20 \%(7)$ \\
\hline $40-49$ & $15 \%(5)$ \\
\hline $50-59$ & $32 \%(11)$ \\
\hline 60 or above & $6 \%(2)$ \\
\hline Missing & $12 \%(4)$ \\
\hline \multicolumn{2}{|l|}{ Gender } \\
\hline Female & $100 \%(34)$ \\
\hline Male & $0 \%(0)$ \\
\hline \multicolumn{2}{|l|}{ Race/Ethnicity } \\
\hline Hispanic & $3 \%(1)$ \\
\hline White & $53 \%(18)$ \\
\hline Black African-American & $35 \%(12)$ \\
\hline Missing & $9 \%(3)$ \\
\hline
\end{tabular}




\section{RESULTS}

Demographic characteristics of participants are shown in Table 1. From these interviews, we assessed HCPs' knowledge of terminology related to the epidemiology of BBC. We also extracted $\mathrm{HCP}$-reported factors that influence how and what they communicated to patients regarding breast cancer.

3.1 Provider knowledge about BBC was limited. Only two of the $24 \mathrm{HCPs}$ who were queried about the use of terminology (this group excludes physicians and PAs) said they had heard the term BBC and five participants were familiar with the term "triple-negative" breast cancer. Even those few who were familiar with these terms did not report using them with patients, and several noted that although they had heard the terms, they were not comfortable defining or using them. Three participants (1 nurse and 2 health educators) correctly noted that these types of cancer are more prevalent in African-American populations.

\subsection{Provider and patient} misinformation about cancer contributed to poor communication of breast cancer, especially subtype, and risk. Scientific misinformation about breast cancer risk was evident, primarily in two ways. First, physicians and PAs reported patient confusion about causes of breast cancer and described spending time addressing beliefs, answering questions, and explaining breast cancer risk factors. For example:

PA: Some [patients] just have old wives tales and myths that get perpetuated into the community...you know, if you fall and hurt your breasts you'll get breast cancer.

Second, some of the self-reported comments made by the HCPs to patients also reflected scientific misinformation, espe- cially related to the relevance of family history for breast cancer risk. For example:

PA: A lot of women think because their sister's cousin had breast cancer that they're at an increased risk. They don't realize that it's your mother and grandmother that increases your risks more so than your father's mother or father's sister.

Given that family history-based risk models specify only the number of firstdegree relatives, the notion that maternal vs. paternal relatives confer different risk is inconsistent with current risk models. ${ }^{25}$

\subsection{Factors Influencing HCP} communication about cancer risk.

Patient health priorities. The opportunity for HCPs to discuss cancer risk during patient visits was often determined by the patient's health priorities. Across the board, HCPs said they tended to focus on weight management, diet, exercise, heart disease, and diabetes as pressing patient health issues. Cancer was a lower priority, especially for patients that presented with more urgent problems, such as poorly managed diabetes or hypertension. Providers sometimes discussed pap smears and mammograms as important preventive tests, but noted that on any given visit, other concerns were a priority and cancer prevention was typically the focus at only one visit per year. For example:

Health educator: There are way too many other problems. We're so busy putting out fires that are right there in front of us. Breast cancer ... it gets ignored because the fire drill is happening right now, and they have to do something about that.

Several HCPs expressed that while breast cancer was not a primary focus, it 
should be included in the scope of addressing women's overall health.

Patient fears. RNs and NPs discussed patient fear as a primary barrier to communicating breast cancer risk. They believed some patients feared discovering if they had breast cancer because "They've seen women go through breast cancer and not have very positive outcomes." HCPs felt that many patients thought if they went looking for cancer then they would subsequently find it, and that they were afraid of poor outcomes. Other HCPs pointed out that because patients feared cancer, they relied on their spiritual beliefs with respect to fate in order to dismiss active discussions about their health. Many HCPs stated that patients believed that an external locus of control (i.e. God or fate) would determine whether they would get cancer, and that it was something beyond their control.

Patient characteristics (age, race, socioeconomic status). Patient age, race, and socioeconomic status were cues for whether and how HCPs discussed breast cancer.

Age. Physicians and PAs focused on cancer screening, heart disease, and diabetes with their older patients, and conversely focused on reproductive issues including birth control, new motherhood, and sexually transmitted diseases with younger patients. HCPs also described older women as asking more questions and having more knowledge about breast cancer, in part because of life experiences and personal connections to the disease.

Race. When asked if HCPs relayed information differently to patients as a result of their race/ethnicity, most HCPs stated that they did not. Nine of the HCPs acknowledged that they serve a large number of minority patients, and a subset of them regularly convey to African-American patients their higher rate of breast cancer mortality as compared to White women. However, discussion of other diseases that frequently affect African-Americans, such as diabetes or heart disease, often took precedence in a short visit. For example:

Physician: People who are AfricanAmerican have an increased risk of diabetes and hypertension, so I may focus more on that and use their race as a tool for education.

Socioeconomic status (SES). Several PAs described tailoring their conversations with patients based on their perceptions of the patient's education or SES, with perceptions of lower education and/or lower SES corresponding to more limited discussion of risk in some cases. RNs and NPs cited low SES as a barrier to recommending online references for additional information to patients, recognizing that this group of women may not have access to internet services. Provider comments highlighted how perceived SES determined communication strategies, for example:

PA: If I've got the poor Black farmer, I'm going to talk to him very differently than my White master's degree person that expects me to speak properly to him... when I see a patient, I don't automatically say, "oh, okay, I have to now use a different term for him." It's within that communication and the context of our talk that we change.

Physician: A lot of times [patients of a low SES] don't ask as many questions versus an educated person who might ask a lot of questions... I feel like sometimes you spend a lot of time with the low SES people more doing social education... instead of answering questions, it's like you're picking and choosing which catastrophe you're going to focus on. 
Insufficient clinic time. Most HCPs complained that lack of time was a barrier to giving patients adequate information about breast cancer risk. Some nurses, in comparing patient education in the clinical setting and in traditional community outreach settings, noted that there is only enough time to address specific medical questions, leaving no allotted time to discuss breast cancer risk, prevention, screening, or sub-types with at-risk patients.

\subsection{Content of HCPs' communication about cancer risk}

Varied cancer risk factors were discussed when time allowed. When discussing breast cancer risk factors with patients, physicians addressed family history, estrogen (i.e., hormone replacement therapy, hormonal contraception, early menarche, late menopause), high fat diet, high alcohol intake, and smoking. Several physicians mentioned teaching that increased parity and breastfeeding are protective factors. Some physicians also mentioned discussing weight control and obesity as modifiable risk factors with patients, for example:

Physician: I don't think I have a lifestyle recommendation for breast health...the thing is, the diet for breast health is the same as for cancer, diabetes, and heart disease.

In their discussions regarding breast cancer risk, RNs and NPs also mentioned diet, exercise, smoking, body mass index, age, breast abnormalities, and general preventative health care. Family history was described as both a potential risk factor and as the catalyst for starting a discussion about breast cancer, with nurses noting that patients often asked questions about breast cancer as a result of a family member of the patient receiving the diagnosis.
National breast screening guidelines did not discourage conversations about breast self-exams. The 2009 United States Preventive Services Task Force (USPSTF) recommendations on screening for breast cancer suggested that breast selfexaminations (BSEs) were not necessary and that mammograms are recommended biannually for women age 50-74 who are not at high risk. These new recommendations had limited influence on how HCPs addressed screening with women 40-50 years old. Twenty-three of the 34 HCPs said they continue to mention and encourage BSE to patients. While some HCPs stated that they were proponents of BSE performance by patients, others referred to the practice as "controversial" and the remainder did not mention it or recommended against it. Several HCPs described focusing instead on the physical changes that women should look for (e.g. skin changes, nipple changes, discharge), emphasizing that "it's still important for a woman to know her body." For mammograms, physicians said they typically still recommended starting at age 40 and repeating every two years; however, if patients did not want to do it, they would not pressure them.

Use of cancer terminology dependent on an estimation of the patients' ability to comprehend. When discussing cancer risk, some HCPs emphasized that the use of technical terms was an important part of the conversation, and served the purpose of exposing patients to the full scope of a health issue. However, a majority of HCPs noted their concerns, hesitations, and avoidance of using technical terms with patients. For example, some felt that "people just sign off" when providers use technical terms or that it might make patients feel "inferior". Others felt that using technical terms would cause confusion and either stifle patient interest or conversely raise more questions, consequently consuming the 
already limited time that providers have to spend with each patient. For example:

PA: It's a pet peeve when a provider is talking to someone who doesn't have any medical knowledge and they throw these big words at them. [Patients] don't understand, and they're not going to ask because they're almost embarrassed to do it.

Some providers used the technical terms with certain caveats; they take conversational cues from the patient and follow up with a layman's definition or metaphor to help broaden the patient's understanding. RNs noted that their use of technical terms depended upon whether they believed their patients could understand it, with some specifically citing patients' lack of education as a reason for non-use. The health educators and dieticians also described both the avoidance of using technical terms with patients, and also their efforts to make challenging words more accessible.

\section{DISCUSSION}

This study was designed to explore HCPs' accounts of factors that influence the communication of breast cancer risk information. With the exception of one nurse, all of the HCPs in our study were general practitioners, not specializing in cancer care. We selected community practitioners because the patient population of interest, African-American women, were more likely to have contact with primary care providers than specialists. Our intention was to characterize conversations about breast cancer prevention in the context of primary care visits.

For breast cancer broadly, lack of time and knowledge among primary care physicians have been documented as a failure to appropriately identify and refer high-risk women. ${ }^{26,27}$ Our findings demonstrate that when the topic of cancer did arise, HCPs strongly emphasized screening but spent little time discussing modifiable risk factors. Barriers to communicating breast cancer risk included limited time, assessments of patient receptivity, patient characteristics, and scientific misinformation. Scientific misinformation was particularly noteworthy in relation to family history and USPSTF guidelines, as has been reported by others, ${ }^{28-}$ ${ }^{30}$ underscoring the need to better educate HCPs about non-modifiable (genetic) versus modifiable risk factors.

Nurses, nurse practitioners, health educators, and dietitians were queried regarding $\mathrm{BBC}$, given this knowledge has been previously characterized among physicians. ${ }^{24}$ Importantly, only a minority of HCPs knew of the BBC subtype. Given the lack of risk factor education regarding breast cancer overall, it is not surprising that specific subtypes of breast cancer were rarely discussed in primary care settings. While many providers are aware of mortality disparities in breast cancer that can be linked to these subtypes, future efforts to educate HCPs should build upon this awareness by presenting new research that links these disparities to incidence of specific breast cancer subtypes. ${ }^{31,32}$ Recent data suggests that beyond querying patients about family history of breast cancer, asking patients about the pathologic characteristics of their family member's breast cancer can help improve genetic risk assessment. ${ }^{33}$ Efforts to provide information to HCPs are important in disseminating prevention strategies for BBC.

Two recurrent themes that influenced HCP-patient discussion of breast cancer risk were patients' emotional reactions and their spiritual beliefs. Patient fear was characterized as a deterrent to discussions about breast cancer prevention and risk, with some HCPs believing that African-American 
women's perceptions of cancer as uniformly deadly prevented them from wanting to discuss it. Additionally, HCPs believed that some women's religious perspective impeded discussions about breast cancer risk and prevention, specifically when women believed that the future was predetermined or in "God's control," and that their action or inaction would not influence outcomes. Similar views regarding fatalism, spiritual beliefs and religiosity influencing AfricanAmerican women's decisions about cancer prevention and control have been reported. ${ }^{34-}$ 37 Issues regarding spirituality and health continue to underscore the importance of faith as a prominent subject in AfricanAmerican lives. When considering interventions to communicate risk, women's emotional appraisal and spiritual beliefs about cancer risk should be reflected in the content and presentation of messages targeting African-American women.

Another important challenge noted was that patients who were perceived as lower SES by HCPs received less comprehensive information. HCPs reported that they simplified information given and relayed less complicated information because they perceived these patients as not having sufficient knowledge about their own health, unable to ask the right questions, and incapable of understanding cancer risk in the context of other issues in their lives that might create a more complicated picture. Indeed, it has been documented that patients with lower literacy levels tend to ask fewer questions and make fewer requests for information, and our HCPs' comments support these reports. ${ }^{38,39}$ Often, low SES patients are minority patients. ${ }^{40}$ In the United States, the quality of health care for minority patients compared to Whites has been extensively documented, underscoring that minority patients receive lower quality interpersonal care. ${ }^{41}$ Minorities rate the quality of interpersonal care by providers more negatively than Whites, ${ }^{42,43}$ and have reported receiving less information and fewer opportunities to participate in decision-making during medical appointments. ${ }^{44}$ African-Americans also report having more unmet communication needs, receiving less information for decision-making, and poor satisfaction with health care compared to Whites ${ }^{45-47}$ factors that adversely affect patient health outcomes.

Similar assumptions were made by HCPs in our study regarding patients' ability to understand technical terms, such as breast cancer subtypes. Information and tools appropriate for women with low literacy are important for improving communication with this at-risk group. Mortality disparities cannot be fully addressed if women of all educational levels cannot be reached with existing prevention messages.

With regard to cancer prevention, HCPs displayed a substantial knowledge of risk factors for breast cancer overall. However, there was also evidence of some misinformation among a subset of PAs and RNs. This finding suggests that with the rapidly changing nature of information in cancer research, especially cancer subtypes, HCPs need support for receiving and communicating up to date information. Although the USPTF guidelines were perceived by some HCPs as confusing for patients, many HCPs followed these guidelines but continued to promote BSE. Physicians emphasized mammography, which is important because physician recommendation is a major factor determining adherence to mammography.

HCPs are fundamentally important to effective prevention, and effective communication is a critical factor in patient decision-making. ${ }^{48}$ However, time is limited in the primary care setting, and a number of factors impeding discussions of relevant patient information that may affect 
preventative health behaviors have been identified, including low frequency of patient requests for breast cancer-specific information, patients' culturally and religiously-based reservations, and provider misinformation. Thus, a two-pronged strategy that provides tools to HCPs for easy access to (1) patient-centered information that can be directly disseminated to women and (2) more detailed, up to date scientific information for HCPs is needed. Additionally, settings outside of clinical practice, such as community and faith-based events, should also be considered as avenues to raise awareness among this population about breast cancer risk and prevention strategies, including specific BBCassociated risks. While many of the barriers identified in this study have been reported previously, ${ }^{49,50}$ increased awareness of the gaps in patient-provider communication is important for improved communication.

Limitations. While our research provides insight into the perspectives of HCP communication about breast cancer, there are some limitations. Our study is based on a convenience sample of HCPs, which may not be representative of primary care HCPs. All providers were women therefore potential gender differences could not be noted. We selected our sampling strategy to ensure a variety of HCPs in rural and urban communities were represented, and to ensure an understanding of diverse communication issues related to breast cancer. However, these HCPs represent various health care sectors and as such have different levels of training, roles, and knowledge of breast cancer. Therefore, while understanding the prominent themes presented, these results should be interpreted with caution as they may not reflect issues specific to a particular medical group.

\section{CONCLUSION}

The present study underscores that HCP communication with patients remains an important nexus for sharing emerging science, and continued focus on novel strategies for accurate and effective communication about general breast cancer risk, as well as specific subtypes, is needed. The identified barriers and facilitators to communication provide important implications for developing effective interventions to increase awareness and, ultimately, improve breast cancer outcomes.

\section{Acknowledgements}

This research was supported by a grant provided by the National Cancer Institute and National Institute of Environmental Health Sciences (National Institutes of Health) Breast Cancer and the Environment Research Program, grant U01 ES019472 and by funds from Avon Foundation, a Nutrition Obesity Research Center (grant no.P30DK056350), the UNC Center for Environmental Health and Susceptibility (grant no. P30ES010126), and a Breast SPORE (grant no. P50-CA058223). We thank Nicole Wilkes for help with data collection. We are grateful to our participants for their time and contribution to this work. LM was funded by UNC University Cancer Research Fund. The authors have no funding or conflicts of interest to disclose. 


\section{REFERENCES}

1. American Cancer Society. 2014. Cancer Facts \& Figures 2013. Atlanta: American Cancer Society.

2. Petracci, E., Decarli, A., Schairer, C., et al. Risk factor modification and projections of absolute breast cancer risk. J Natl Cancer Inst. 2011.

3. Zhang, S.M., Lee, I.M., Manson, J.E., et al. Alcohol consumption and breast cancer risk in the Women's Health Study. Am J Epidemiol. 2007;165(6):667-76.

4. Wu, Y., Zhang, D., \& Kang, S. Physical activity and risk of breast cancer: a meta-analysis of prospective studies. Breast Cancer Res Treat. 2013;137(3), 869-882.

5. Cuzick, J., DeCensi, A., Arun, B., et al. Preventive therapy for breast cancer: a consensus statement. Lancet Oncol. 2011;12(5) , 496-503.

6. Herman, J. D., \& Herman, S. M. Women's understanding of personal breast cancer risk: Does ethnicity matter? In ASCO Annual Meeting Proceedings, 2013;(Vol. 31, No. 26_suppl, p. 4).

7. Manary, M. P., Boulding, W., Staelin, R., $\&$ et al. The patient experience and health outcomes. $N$ Engl J Med. 2013;368(3), 201-203.

8. Cooper, L.A., and Barnes, D. Journey to eliminating health care disparities: the urgency of affirming values within our patients, profession, health care system, and society. Arch Intern Med. 2012;172, 1667-9.

9. Ledford, C. J., Ledford, C. C., \& Childress, M. A. Extending Physician ReACH: Influencing patient activation and behavior through multichannel physician communication. Patient Educ Couns. 2013;91(1), 72-78.

10. Zolnierek, Kelly B. Haskard, and M. Robin DiMatteo. "Physician communication and patient adherence to treatment: a metaanalysis." Med Care. 2009;47.8:826.

11. Thorne, S.E., Bultz, B.D., Baile, W.F., et al. Is there a cost to poor communication in cancer care?: a critical review of the literature. Psychooncology. 2005;14, 875-84.

12. Weber, K.M., Solomon, D.H., and Meyer, B.J. A qualitative study of breast cancer treatment decisions: evidence for five decision-making styles. Health Commun. 2013;28, 408-21.

13. Street, R. L., Makoul, G., Arora, N. K., et al. How does communication heal? Pathways linking clinician-patient communication to health outcomes. Patient Educ Couns. 2009;74(3), 295-301.

14. Penner, L. A., Blair, I. V., Albrecht, T. L., et al. Reducing Racial Health Care Disparities A Social Psychological Analysis. Policy Insights Behav Brai n Sci. 1(1), 2014;204-212.

15. Siminoff, L. A., Graham, G. C., \& Gordon, N. H. Cancer communication patterns and the influence of patient characteristics: disparities in information-giving and affective behaviors. Patient Educ Couns. 2006;62(3), 355-360.

16. Blair, I.V., Steiner, J.F., Fairclough, D.L., et al. Clinicians' implicit 
ethnic/racial bias and perceptions of care among black and Latino patients. Ann Fam Med. 2013;11, 43-52.

17. Bao, Y., Fox, S.A., and Escarce, J.J. . Socioeconomic and racial/ethnic differences in the discussion of cancer screening: "between-" versus "within-" physician differences. Health Serv Res. 2007;42 (3 Pt 1), 950-70.

18. Dietze, E. C., Sistrunk, C., MirandaCarboni, G., et al. Triple-negative breast cancer in African-American women: disparities versus biology. Nat Rev Cancer. 2015;15(4), 248254.

19. Neilson, H. K., Friedenreich, C. M., Brockton, N. T., et al. Physical activity and postmenopausal breast cancer: proposed biologic mechanisms and areas for future research. Cancer Epidemiol Biomarkers Prev. 2009;18(1), 11-27.

20. Flegal, K. M., Carroll, M. D., Kit, B. K., et al. Prevalence of obesity and trends in the distribution of body mass index among US adults, 19992010. JAMA. 2012;307(5), 491-497.

21. Groth, S. W., Holland, M. L., Kitzman, H., et al. Gestational weight gain of pregnant African-American adolescents affects body mass index 18 years later. J Obstet Gynecol Neonatal Nurs. 2013;42(5), 541-550.

22. Centers for Disease Control and Prevention (CDC). Progress in increasing breastfeeding and reducing racial/ethnic differencesUnited States, 2000-2008 births. MMWR Morb Mortal Wkly Rep 2013;62(5), 77.
23. Partridge, A.H., Elmore, J.G., Saslow, D., et al. Challenges in ductal carcinoma in situ risk communication and decisionmaking: report from an American Cancer Society and National Cancer Institute workshop. CA Cancer J Clin. 2012;62, 203-10.

24. Guest G, MacQueen KM, Namey EE. Applied Thematic Analysis. Los Angeles (CA): Sage Publications. 2012.

25. Amir, E., Freedman, O. C., Seruga, B., et al. Assessing women at high risk of breast cancer: a review of risk assessment models. J Natl Cancer Inst. 2010;102(10), 680691.

26. Meissner, H. I., Klabunde, C. N., Breen, $\mathrm{N}$., et al. Breast and colorectal cancer screening: US primary care physicians' reports of barriers. Am J Prev Med. 2012;43(6), 584-589.

27. Yarnall, K. S., Pollak, K. I., Østbye, T., et al. Primary care: is there enough time for prevention? Am J Public Health. 2003;93(4), 635-641.

28. Haas, J. S., Sprague, B. L., Klabunde, C. N., et al. Provider attitudes and screening practices following changes in breast and cervical cancer screening guidelines. J Gen Intern Med. 2015;1-8.

29. McCuaig, J.M., Greenwood, C.M., Shuman, C., et al. Breast and ovarian cancer: the forgotten paternal contribution. J Genet Couns. 2011;20, 442-9.

30. Burke, W., Culver, J., Pinsky, L., et al. Genetic assessment of breast cancer risk in primary care practice. Am J Med Genet A. 2009;149A, 349-56. 
31. Wheeler, S. B., Reeder-Hayes, K. E., \& Carey, L. A. Disparities in breast cancer treatment and outcomes: biological, social, and health system determinants and opportunities for research. Oncologist. 2013;18(9), 986-993.

32. O'Brien, K.M., Cole, S.R., Tse, C.K., et al. Intrinsic breast tumor subtypes, race, and long-term survival in the Carolina Breast Cancer Study. Clin Cancer Res. 2010;16, 6100-10.

33. Mavaddat, N., Rebbeck, T.R., Lakhani, S.R., et al. Incorporating tumour pathology information into breast cancer risk prediction algorithms. Breast Cancer Res. 2010;12(3):R28.

34. Sterba, K. R., Burris, J. L., Heiney, S. P., et al. "We both just trusted and leaned on the Lord": a qualitative study of religiousness and spirituality among African-American breast cancer survivors and their caregivers. Qual Life Res. 2014;23(7), 1909-1920.

35. Gullatte, M. M., Brawley, O., Kinney, A., et al. Religiosity, spirituality, and cancer fatalism beliefs on delay in breast cancer diagnosis in AfricanAmerican women. J Relig Health. 2010;49(1), 62-72.

36. Sheppard, V. B., Adams, I. F., Lamdan, R., et al. The role of patient-provider communication for black women making decisions about breast cancer treatment. Psychooncology.

2011;20(12), 1309-1316.

37. Conway-Phillips, R., and Janusek, L. Influence of sense of coherence, spirituality, social support and health perception on breast cancer screening motivation and behaviors in AfricanAmerican women. ABNF J. 2014;25(3), 72.
38. Aboumatar, H. J., Carson, K. A., Beach, M. C., et al. The impact of health literacy on desire for participation in healthcare, medical visit communication, and patient reported outcomes among patients with hypertension. J Gen Intern Med. 2013;28(11), 1469-1476.

39. Mazor, K. M., Rubin, D. L., Roblin, D. W., et al. Health literacy-listening skill and patient questions following cancer prevention and screening discussions. Health Expect. 2015.

40. DeNavas-Walt, Carmen, Bernadette D. Proctor, and Jessica C. Smith. Income and poverty in the United States: 2013. United States Census Bureau, 2014.

41. Shavers, V. L., Klein, W. M., \& Fagan, P. Research on race/ethnicity and health care discrimination: where we are and where we need to go. Am $\mathbf{J}$ Public Health. 2012;102(5), 930-932.

42. Cooper, L. A., Roter, D. L., Carson, K. A., et al. The associations of clinicians' implicit attitudes about race with medical visit communication and patient ratings of interpersonal care. Am J Public Health. 2012;102(5), 979-987.

43. Quach, T., Nuru-Jeter, A., Morris, P., et al. Experiences and perceptions of medical discrimination among a multiethnic sample of breast cancer patients in the Greater San Francisco Bay Area, California. Am J Public Health. 2012;102, 1027-34.

44. Song, L., Hamilton, J.B., and Moore, A.D. Patient-healthcare provider communication: perspectives of African-American cancer patients. Health Psychol. 2012;31, 539-47. 
45. Lin, M. Y., \& Kressin, N. R. Race/ethnicity and Americans' experiences with treatment decision making. Patient Educ Couns. 2015.

46. Williams, S. W., Hanson, L. C., Boyd, C., et al. Communication, decision making, and cancer: what AfricanAmericans want physicians to know. J Palliat Med. 2008;11(9), 1221-1226.

47. Thornton, R. L. J., Powe, N. R., Roter, D., et al. Patient-physician social concordance, medical visit communication and patients' perceptions of health care quality. Patient Educ Couns. 2011;85(3), e201-e208.
48. Epstein, R.M., and Street, R.L.J. PatientCentered Communication in Cancer Care: Promoting Healing and Reducing Suffering. J Natl Cancer Inst, NIH Publication: Bethesda. 2007.

49. Yarnall, Kimberly SH, et al. "Primary Care: Is there Enough Time for Prevention?" Am J Public Health. 93.4 (2003): 635-641.

50. Davis, Terry C., et al. "Health Literacy and Cancer Communication." CA Cancer J Clin. 52.3 (2002): 134-149. 